\title{
Flow compressibility effects around an open-wheel racing car
}

J. Keogh

j_keogh @live.com.au

G. Doig

g.doig@unsw.edu.au

School of Mechanical and Manufacturing Engineering

The University of New South Wales

Sydney, New South Wales

Australia

\section{S. Diasinos}

sammy.diasinos@mq.edu.au

Macquarie University

North Ryde

New South Wales

Australia

\section{ABSTRACT}

A numerical investigation has been conducted into the influence of flow compressibility effects around an open-wheeled racing car. A geometry was created to comply with $2012 \mathrm{~F} 1$ regulations. Incompressible and compressible CFD simulations were compared - firstly with models which maintained Reynolds number as Mach number increased, and secondly allowing Mach number and Reynolds number to increase together as they would on track. Results demonstrated significant changes to predicted aerodynamic performance even below Mach $0 \cdot 15$. While the full car coefficients differed by a few percent, individual components (particularly the rear wheels and the floor/ diffuser area) showed discrepancies of over 10\% at higher Mach numbers when compressible and incompressible predictions were compared. Components in close ground proximity were most affected due to the ground effect. The additional computational expense required for the more physically-realistic compressible simulations would therefore be an additional consideration when seeking to obtain maximum accuracy even at low freestream Mach numbers. 


\section{NOMENCLATURE}

$c \quad$ front wing chord $(\mathrm{m})$

$C_{D} \quad$ coefficient of force in the direction aligned with freestream, based on frontal area

$C_{L} \quad$ coefficient of force in the direction perpendicular to freestream, based on frontal area (negative lift)

$C_{M} \quad$ coefficient of moment

$C_{P} \quad$ coefficient of pressure

$D \quad$ drag force $(\mathrm{N})$

$h \quad$ minimum height above the ground $(\mathrm{m})$

$l \quad$ total car length $(\mathrm{m})$

$L \quad$ lift force (N)

$\dot{\mathrm{m}} \quad$ mass flux $\left(\mathrm{kgm}^{-2} \mathrm{~s}^{-1}\right)$

M Mach number

$U_{\infty} \quad$ vehicle speed $\left(\mathrm{ms}^{-1}\right)$

$x \quad$ Cartesian co-ordinate aligned with freestream, positive in the downstream direction $(\mathrm{m})$

$y \quad$ Cartesian co-ordinate positive in the starboard direction $(\mathrm{m})$

$z \quad$ Cartesian co-ordinate normal to freestream, directed upwards (m)

$k \quad$ turbulent kinetic energy $\left(\mathrm{m}^{2} / \mathrm{s}^{2}\right)$

$\rho$ density $\left(\mathrm{kg} / \mathrm{m}^{3}\right)$

$\varepsilon \quad$ turbulent dissipation $\left(\mathrm{m}^{2} / \mathrm{s}^{3}\right)$

$\omega \quad$ specific dissipation rate $\left(s^{-1}\right)$

\section{Abbreviations}

CAD Computer-aided design

CFD Computational Fluid Dynamics

DES Detached Eddy Simulation

F1 Formula One

FIA Fédération Internationale de l'Automobile

GCI Grid Convergence Index

SA Spalart-Allmaras

SST Shear Stress Transport

RANS Reynolds-Averaged Navier Stokes

\subsection{INTRODUCTION}

There are several key considerations when designing an aerodynamic package for an open-wheeled racing car. The design has to provide the maximum amount of downforce, or negative lift, for a specified drag target. Downforce increases the mechanical grip of the tyres and allows increased levels of acceleration and cornering speed to be achieved. The car has to be balanced so as to make it predictable and drivable ${ }^{(1-3)}$. There is a high level of importance placed on the aerodynamicist having a comprehensive understanding to be able to optimise performance ${ }^{(4)}$. The complexity of aerodynamic design of such vehicles has grown considerably in the last decade thanks in part to the increased ability of teams to use Computational Fluid Dynamics (CFD); the volume and variety of testing that CFD facilitates would be both economically and physically impossible using real-world approaches. 
Three methods of aerodynamic testing currently exist: CFD, wind tunnel and track testing. CFD is the primary tool of the aerodynamicist in analysing any new design. Perfect correlation does not exist between the three methods of aerodynamic analysis for a number of reasons ${ }^{(5)}$. CFD simulations assume an ideal controlled environment and in industry usually consist of Reynoldsaveraged, incompressible, steady-state solutions. This assumes that flow behaviour will remain steady, independent of time, and that density changes in the air will be negligible within the expected speed range - neither of which is possible in the real world. Wind tunnels have issues of scaling (a maximum $60 \%$ model is permissible under regulations ${ }^{(6)}$, with some teams using $50 \%$ scale) and require intrusive devices to both hold the car in place and take measurements. They are further limited with regulations dictating the maximum speed at which teams are able to test (currently $50 \mathrm{~ms}^{-1}$, which equates to $30 \mathrm{~ms}^{-1}$ at full scale for the same Reynolds number). This is a speed that might only be reached momentarily during cornering - certainly not the straight line condition the wind tunnel is representative of. On-track testing is difficult, pressure measurements can be taken over the surface of the vehicle and in some instances bulky measurement devices will be fitted in an attempt to obtain further information. Ultimately track performance becomes highly dependent on driver consistency and feedback. However, the track is where the performance really matters.

The intricacy of a modern race car requires a very high fidelity simulation to accurately capture all features of the geometry, and this entails great computational expense. Absolute accuracy must be compromised to allow results to be obtained quickly and within the restrictions placed on development resources. The process is a calculated compromise which allows the team to put the best car out on the track while remaining compliant with both regulations and deadlines. Along with the use of the Reynolds-averaged form of the Navier-Stokes equations, a number of further assumptions and simplifications are also made, one being that the density changes in the air flowing around the car will have a negligible influence on predicted aerodynamic performance. This is adopted from a standard assumption within the aeronautical industry that below Mach $0 \cdot 3$, the effects of compressibility around a body can be ignored without significantly compromising the accuracy of the solution. An open wheeled race car will rarely, if ever, reach Mach 0.3 so the assumption of incompressible flow appears reasonable at first, until one considers that the front wing alone can accelerate flow to a local maximum of almost three times the freestream ${ }^{(4)}$.

In Katz's otherwise comprehensive review of racing car aerodynamics, compressibility effects are only mentioned in relation to land speed record cars, which operate in the high-subsonic to low-supersonic regime ${ }^{(2)}$. Zhang et al reviewed the progress made in race-car aerodynamics over the last 30 years and analysed the studies that have been conducted in publicly available literature ${ }^{(4)}$. However, none of the 89 papers reviewed made any specific mention of compressibility effects other than when they were incidental to experiments or numerical solutions. The review highlighted compressibility as an area of race-car aerodynamics yet to receive any thorough investigation within the public domain - the present investigation aims to rectify this.

Doig et $a l^{(7,8)}$, conducting simulations on the front wing in isolation (in section and as a full wing with endplates), investigated whether the assumption of incompressibility might be a source of a percentage of the differences commonly observed when CFD is compared to tunnel data. The results demonstrated that for a single element wing operating in ground effect, the differences between incompressible and compressible simulations can be substantial. Even at Mach $0 \cdot 15(183 \cdot 7 \mathrm{~km} / \mathrm{h})$ the differences found in drag, lift and pitching moment were between two and three percent, with compressible cases predicting a greater flow acceleration and suction peak, and eventually earlier separation. At a freestream Mach number of less than $0 \cdot 4$, the local flow around the wing could become supersonic. The work also demonstrated that a simple compressible correction would not be suitable due to changes in ground proximity sensitivity. The results pointed to a strong need to 
conduct simulations on a full car to understand how all components which produce strong flow gradients would be affected given their interdependencies.

In light of this, the present study aimed to investigate compressibility effects over a simplified full-car model to understand their influence in Reynolds-scaled testing as compared to full-scale CFD, and to examine compressibility in general as both the Mach number and Reynolds number increase naturally together as they would for a car on track.

\subsection{NUMERICAL METHOD}

\subsection{Geometry}

The car model was constructed within the bounds of current Formula One regulations, though is generic enough that its characteristics would be common to most similar categories. A number of measures were also taken to reduce complexity of the model, with the benefit of facilitating clearer observation of components free of excessive interference from others. Basic features of the car are identified in Fig. 1. The geometry is publicly available for future studies. All aerodynamic coefficients described herein are based on a reference frontal area of $1.47 \mathrm{~m}^{2}$ similar to standard practice in Formula One ${ }^{(1)}$.

The car has an overall length of $4,635 \mathrm{~mm}$ at full scale. The maximum width of $1,800 \mathrm{~mm}$ occurs at the widest points of the wheels and the front wing. In between the front and rear wheels the maximum width is $1,400 \mathrm{~mm}$. The maximum height of the car is $950 \mathrm{~mm}$ above the reference plane (the lower central section of the floor, $z=0$ ) and occurs at the highest point of the rear wing endplate and the top of the airbox above the driver's head. A nose-down rake of $1.3^{\circ}$ was applied to the entire body of the car. The front ride height was set at $15 \mathrm{~mm}$, the rear at $75 \mathrm{~mm}$. The driver geometry was kept as a simple helmet-esque shape.

Flow inlets were placed at the front of the sidepods for cooling, and for engine air in the airbox above the driver's head. Exhaust exits were placed at the rear of the sidepods and used a simple circular shape. The outlet for the cooling flow was placed centrally at the rear of the body, in front of the lower main plane on the rear wing assembly. Current cars use a similar arrangement but may also include gills at different locations along the sidepods for additional cooling.

Barge-boards commonly seen on almost all current open-wheel race-cars were removed as a further measure to reduce the complexity of the flowfield. Their high importance in managing flow around the car is acknowledged but the contribution to compressibility effects is likely to be small. Axles, driveshaft and suspension structures were also removed for simplicity.

The car utilises a modified double element front wing taken from literature ${ }^{(9)}$ - the geometry is a T026 main element with a generic flap set at an incidence of $14 \cdot 1^{\circ}$ (Fig. 1(d)). A single element centre section occupies the space of the standardised section required by the FIA (Fédération International de l'Automobile). A basic rectangular $500 \times 215 \times 10 \mathrm{~mm}$ endplate was used. A smaller inner endplate was placed where the wing transitions from the single element to double element section. The chord length is $475 \mathrm{~mm}$ for the double element and $280 \mathrm{~mm}$ for the single element. The wing has a ground clearance of $72.5 \mathrm{~mm}$ at full scale corresponding to a height to chord ratio of $h / c=0 \cdot 153$. A $6 \mathrm{~mm}$ gurney was attached to the trailing edge of the flap, increasing the amount of downforce produced with a minimal drag expense.

The rear wing is a component that has been the subject of very little research in the public domain due to the fact that it is heavily influenced by upstream components. In this instance, the main profile uses two modified s1223 aerofoils with an increased camber. These were derived 

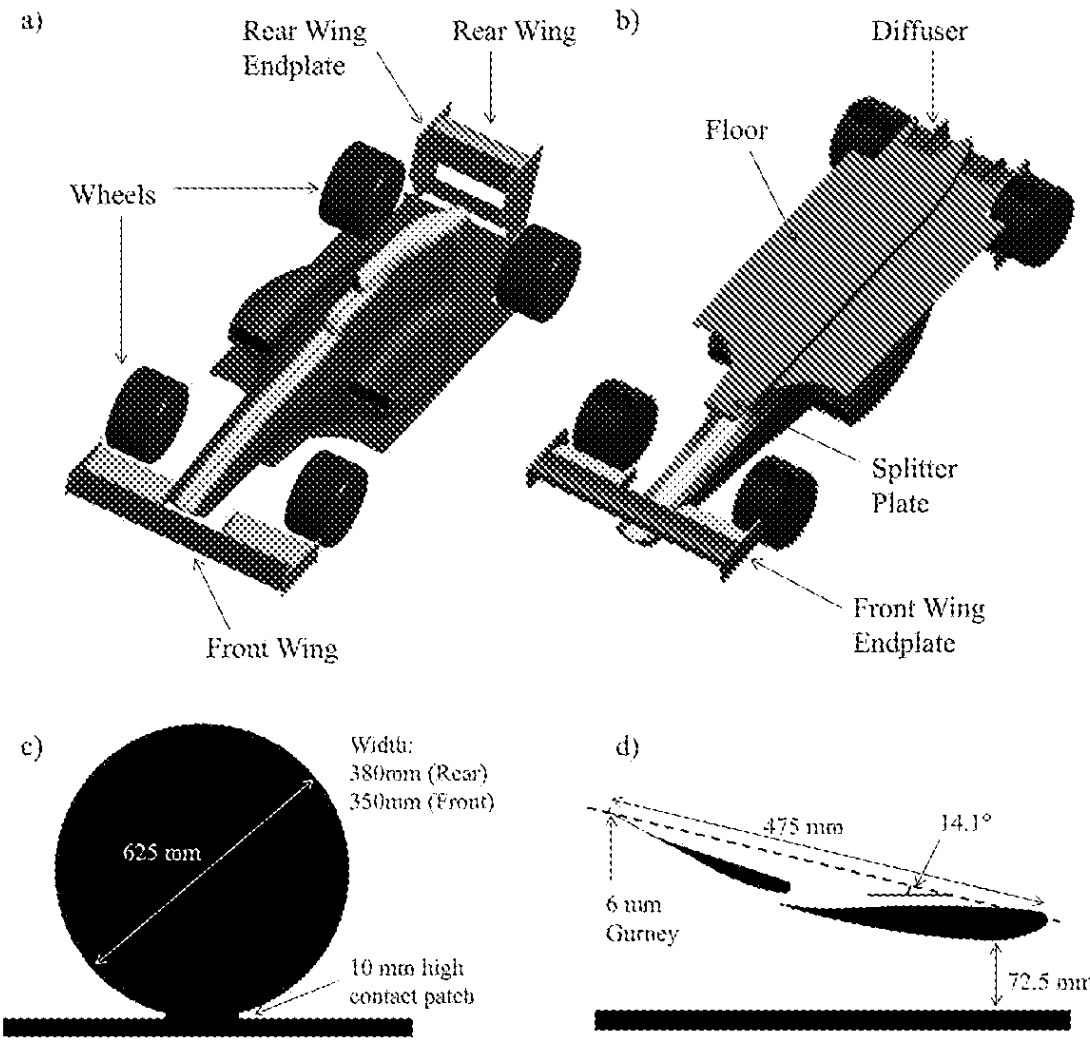

d)
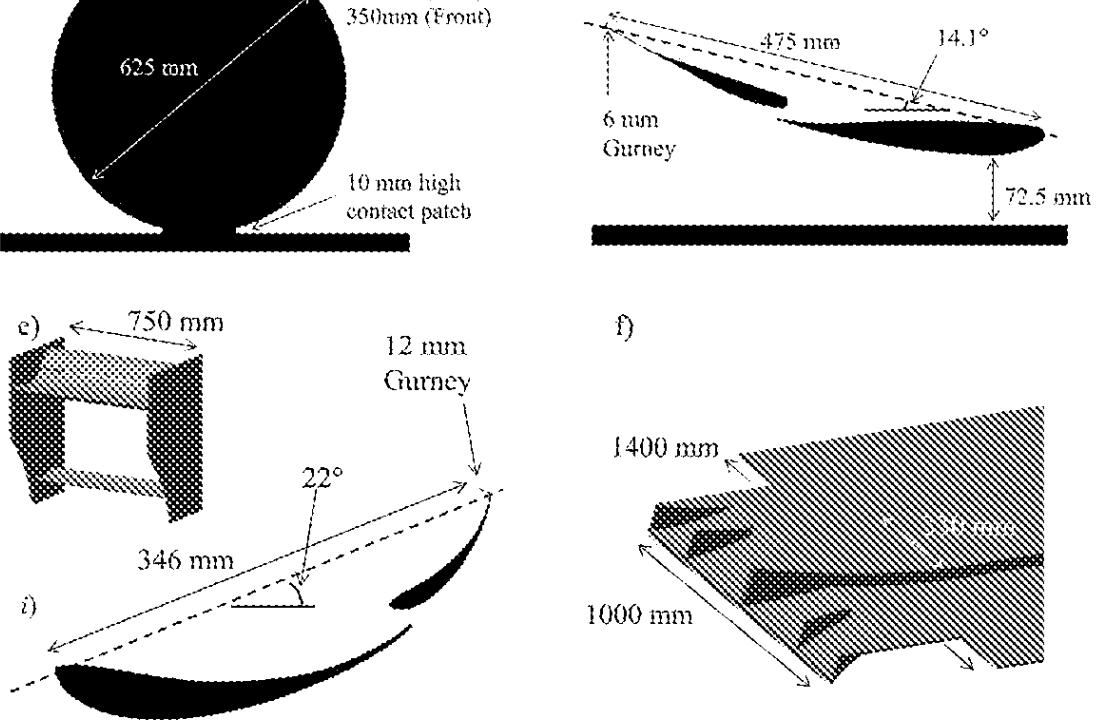

)

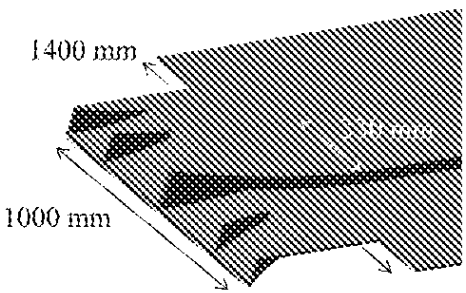

ii)
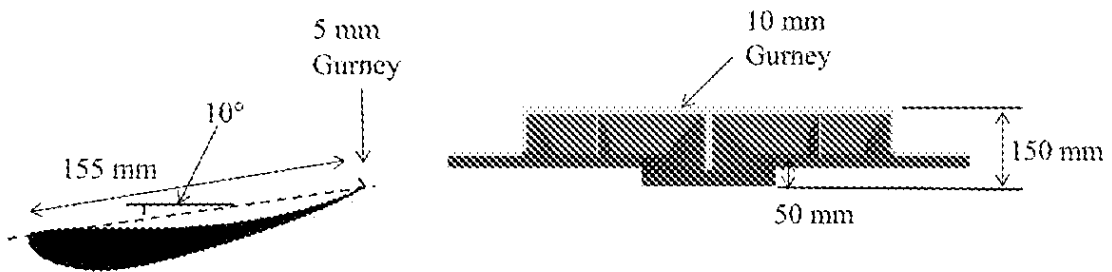

Figure 1. (a) and (b) the car's main components on the upper and lower sides, (c) the wheels, (d) the double-element front wing, (e) the rear wing assembly and (f) the floor/diffuser.

from the airfoil presented by Pakkam in his Master's thesis ${ }^{(10)}$. The s1223 is a common high-lift low-Reynolds number aerofoil that has been used in a number of similar applications. The total chord length was $346 \mathrm{~mm}$ and was set at an incidence of $2^{\circ}$ (Fig. 1(e)). The flap is equipped with a $12 \mathrm{~mm}$ gurney at the trailing edge. The lower main plane uses a standard s1223 aerofoil set at a $10^{\circ}$ incidence with a chord length of $155 \mathrm{~mm}$ using a smaller $5 \mathrm{~mm}$ gurney. A generic $10 \mathrm{~mm}$ thick endplate of simple construction was used. 
A well designed floor and diffuser will allow more efficient production of downforce than any other aerodynamic device on the car. The upswept diffuser section uses a three dimensional expansion, as seen in Fig. 1(f), expanding both vertically and laterally, increasing the acceleration of the air underneath the car and

increasing the production of downforce. A $10 \mathrm{~mm}$ gurney was placed along the uppermost edge of the trailing edge of the diffuser section. A central lower section known as the reference-plane which is $330 \mathrm{~mm}$ in width and sits $50 \mathrm{~mm}$ lower than the main floor section was used as is required by current regulations. At the front of the floor a narrow front splitter was placed, allowing further production of downforce with the flow being accelerated underneath and a high pressure region created above due to the presence of the body of the car. The leading front section across the entire width of the floor also produces negative lift in similar manner. A $50 \mathrm{~mm}$ radius was placed on the narrow front splitter leading edge. A $25 \mathrm{~mm}$ radius was placed on the leading edge of the upper floor plane. A single strake was placed in both sides of the diffuser to direct the flow and produce beneficial vortices.

The wheels were sized to conform to current regulations, sized as shown in Fig. 1(c) and with no hub geometry, which simplifies the flowfield considerably. A $10 \mathrm{~mm}$ high contact patch was placed where the wheel comes into contact with the ground, for ease of meshing and to account somewhat for the deformation that an actual wheel would produce. Diasinos ${ }^{(1)}$ found an increase in the size of the contact patch reduced the wheel wake width due to variation in the separation point over the front of the contact patch. The absence of this geometrical feature in reality and shape change due to deformation of a pneumatic tyre are acknowledged features that would affect the flowfield ${ }^{(12)}$. Further complexities such as brake cooling ducts and turning vanes were not considered.

\subsection{Solver}

ANSYS Fluent 14 was used to produce all the results described in the subsequent sections; it is software commonly used in the motorsport industry. All simulations were run on 64-bit clusters using a pressure-based, coupled solver that has been shown to perform well even for compressible simulations at low Mach numbers ${ }^{(7-8)}$. Only steady-state solutions were considered, and convergence criteria were deemed to be met not only when the mass and momentum scaled-residual errors ceased to change by more than approximately $0.01 \%$ over 1,000 continued iterations, but also when the aerodynamic forces on the body ceased to change by more than $0.01 \%$ over 1,000 further iterations. A second order cell-based upwinding discretisation scheme was applied, as was a standard three-coefficient Sutherland viscosity model for simulations involving compressible (ideal gas) flow. A constant heat capacity was set for all compressible simulations giving a Prandtl-number of 0.74 at the inlet.

\subsection{Boundary conditions}

The domain was constructed to provide a quasi-wind-tunnel environment - the inlet was located 5 car lengths, 1 , upstream, the outlet 101 downstream, the upper and side limits at 31 and 21 respectively. Increasing the size of the domain beyond this was found to produce minimal influence on the aerodynamic coefficients obtained.

For the incompressible simulations the inlet, exhaust exit and cooling flow outlet were all modelled as velocity inlets in the $x$-direction. A turbulence intensity of $0 \cdot 1 \%$ was used, similar to that which might be found in a well-calibrated wind tunnel. For compressible simulations a pressure far-field inlet condition was used to produce identical freestream flow conditions. Inlet freestream density was defined as $1.225 \mathrm{~kg} / \mathrm{m}^{3}$. Again this condition was matched by both types of simulations. The pressure outlet condition with a zero static pressure was used for both types of simulation and was placed at the outlet, cooling flow inlet and engine inlet. 
Previous ground effect studies have demonstrated the importance of a moving ground plane in any ground effect simulation ${ }^{(13)}$; for this reason the ground surface was set as a non-slip boundary with a translational velocity in the positive $x$-direction matching the freestream velocity. All other surfaces were static with the exception of the wheels, given an angular velocity on the surface so the tangential velocity at the surface matched the translational velocity at the ground surface. Due to the symmetrical properties of the geometry a symmetry plane was able to be placed down the centreline. This assumes that the flow will behave identically over both sides of the car and halves the computational expense required.

\subsection{Grid convergence}

A tetrahedral mesh with a concentrated near-wall prism mesh was created in StarCCM+, then imported into Fluent and converted into a polyhedral mesh, as shown in Fig. 2. Cells were concentrated close to the car and in the near wake region, gradually increasing in size further downstream of the car. The approach took advantage of the potential for increased accuracy from polyhedral cells with a slightly reduced memory overhead - despite having 39 million nodes, the finest mesh cell count remained under 10 million.

A grid convergence study was completed considering coarse, medium and fine meshes. The coarse mesh contained $4.08 \times 10^{6}$ cells, at the minimum requirement in order to capture the geometry without producing poor quality cells and excessive faceting. The fine mesh contained $9 \cdot 16 \times 10^{6}$ cells and improved mesh resolution both at the surface and in the wake region. Overall lift and drag values for the car were used as parameters for determining grid convergence from this starting point. While meshes used by Formula One teams typically range between $50-200 \times$ $10^{6}$ cells, this level of resolution was not practical or necessary for the present study.

As can be seen in Fig. 3 the variation in the convergence criteria from the medium to fine grid was less than $4 \%$ for the two parameters being considered. The coarse grid failed to accurately predict negative lift by a very significant margin and was determined to be an unsuitable resolution. Using the method outlined by Roache ${ }^{(14)}$, a Grid Convergence Index (GCI) was calculated to obtain a better indication of the error contained within lift and drag predictions. The GCI is based on the Richardson extrapolation. A safety factor is recommended to accommodate for the Richardson extrapolation not being conservative in maintaining conservation properties. To calculate GCI for the coarse and fine grids the following equations were used:

$$
\mathrm{GCI}^{\text {fine }}=\mathrm{F}_{\mathrm{S}}\left|\mathrm{E}^{\mathrm{fine}}\right| \mathrm{GCI}^{\text {coarse }}=\mathrm{F}_{\mathrm{S}}\left|\mathrm{E}^{\text {coarse }}\right|
$$

where $F_{S}$ is the factor of safety which was set as three due to the number of mesh intervals considered. For the medium to fine grid $r=1 \cdot 21$. Second order accuracy was used for discretisation, giving a theoretical second order error reduction rate $(p=2)$. A least squares fit of the data yielded an order of convergence $p=1 \cdot 54$. This suggested that cell quality, boundary conditions and turbulence modelling may have been responsible for an error reduction rate that was less than two. Using these values the results in Table 1 were calculated, and the fine mesh was chosen to be the most appropriate for all future runs.

\subsection{Front wing validation}

For partial validation of the modelling approach and to assist in selection of an appropriate turbulence model, the full car settings and local mesh resolution were applied to a front wing and isolated wheel for which reliable experimental data existed ${ }^{(15,16)}$. As a result meshes were relatively 

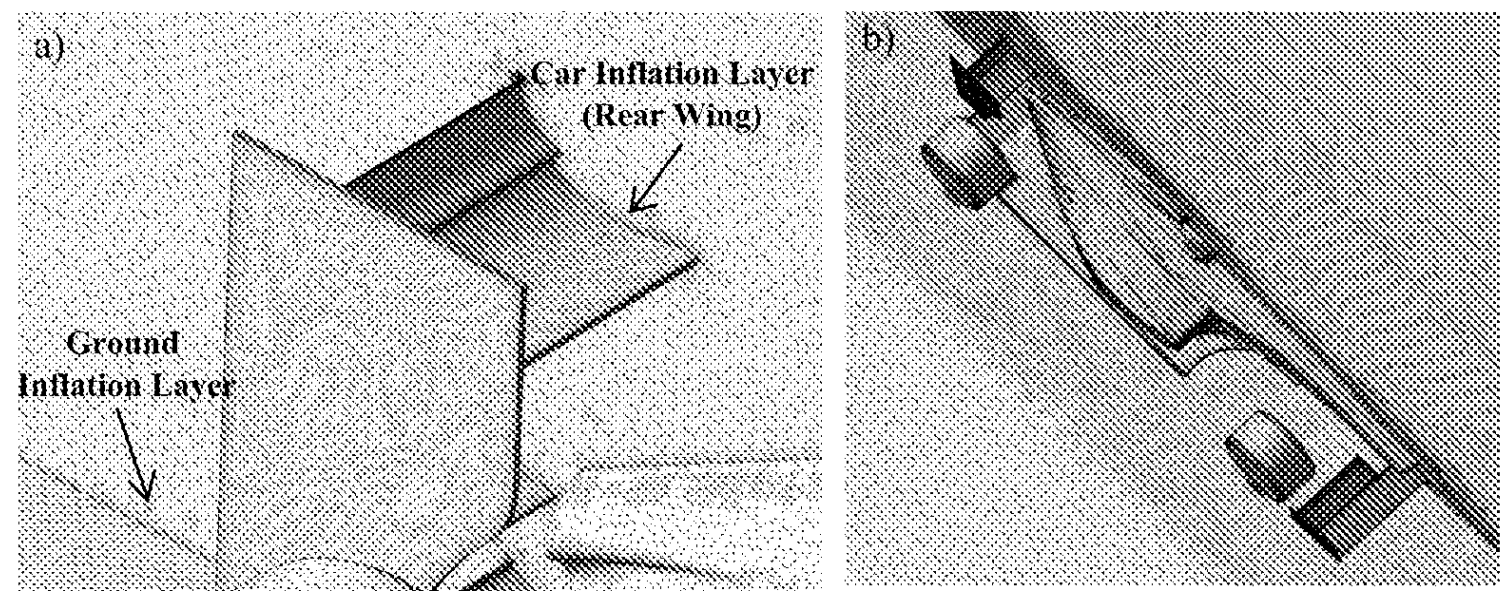

Figure 2. (a) Details of the mesh around the rear wing and on the ground plane, and (b) the mesh on the car surface, ground, and symmetry plane.

coarse in comparison to those which would be used if a detailed study of that component were to be undertaken.

Accurate modelling of a simple front wing was an important step in ensuring that the flow over the rest of the car was representative of reality. Zerihan conducted a series of experiments for a single element T026 wing operating in close ground proximity for his $\mathrm{PhD}$ thesis ${ }^{(17)}$. His experiments were conducted in the Southampton Low Speed wind tunnel, which was reproduced in the CFD wing study here, complete with a proxy of the transition strip (with the fluid around the wing split into laminar and turbulent regions). Other aspects of the model were conducted in accordance with other very similar studies in literature, including those showing that Fluent would produce near-identical results at $30 \mathrm{~ms}^{-1}$ for both compressible and incompressible simulations of the wing at large $h / c$ values (i.e. little ground effect).

The Realisable $k-\varepsilon^{(17)}$, Menter's $k$ - $\omega$ Shear Stress Transport (SST) ${ }^{(19)}$, and Spalart-Allmaras (SA) ${ }^{(20)}$ models were all tested against the results of Zerihan ${ }^{(17)}$, as all have previously been shown to be reasonably capable of reproducing these experimental pressure distributions and force coefficients $^{(21,22)}$. The models were compared against the experimental lift and drag data of Zerihan at $h / c$ $=0 \cdot 090$, as shown in Fig. 4. A good level of correlation was found for the use of the Realisable and SA models in the predicting of both lift and drag. Computational resource availability restricted the mesh resolution in the wake region and was observed to affect the SST model most severely. Good correlation for drag but poor correlation for lift occurred. A $y^{+}$value of around 30 made the standard wall function the appropriate wall model to be used for the Realisable $k-\varepsilon$ model and allowed close correlation for a range of ground clearances. A better $y^{+}$resolution was not maintainable over the whole car for cell quality reasons, and rendered the mesh unsuitable for the enhanced wall treatment employed by the SST model. A reduced suction peak, earlier onset of separation and an inaccurate prediction of the near wake velocity deficit occurred. These factors resulted in the poor performance of the SST model in this instance.

Slight under-prediction of lift and over-prediction of drag was observed for the higher ground clearances, in Fig. 5. The Realisable model was able to give the most accurate prediction of separation point and closest correlation with measure results in the near-wake region, while the SA model was observed to correlate closely with experimental lift values for a range of ground clearances, but had a tendency to over- predict drag and give a poor prediction of the separation point and in the near-wake region. Therefore the Realisable model was deemed preferable overall. 


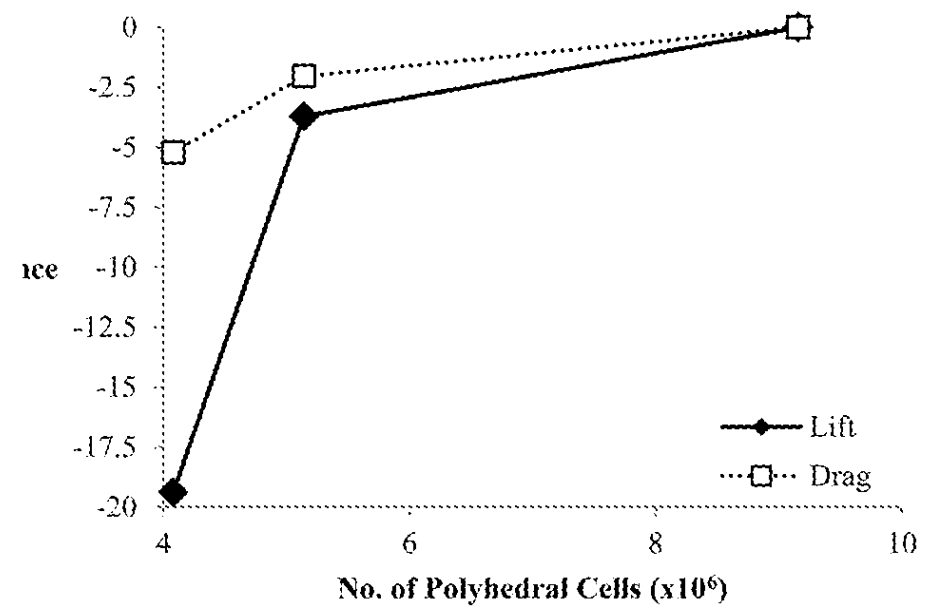

Figure 3. Variance in lift and drag coefficients with increasing mesh resolution.
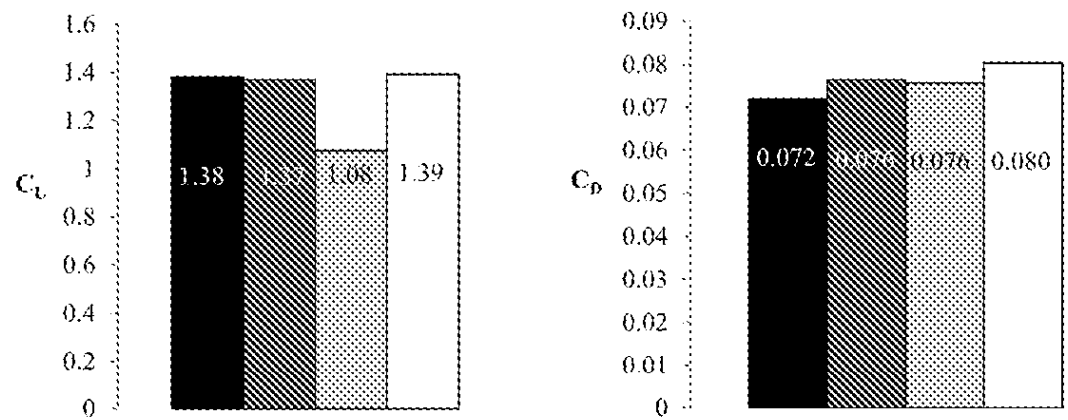

$$
\begin{aligned}
& \text { Exporimental } \\
& \text { Realizablek-8 } \\
& \text { k-coSST } \\
& 0 \text { Spalart-Alumaras }
\end{aligned}
$$

Figure 4. Lift and drag coefficients of the front wing as predicted by different turbulence models.

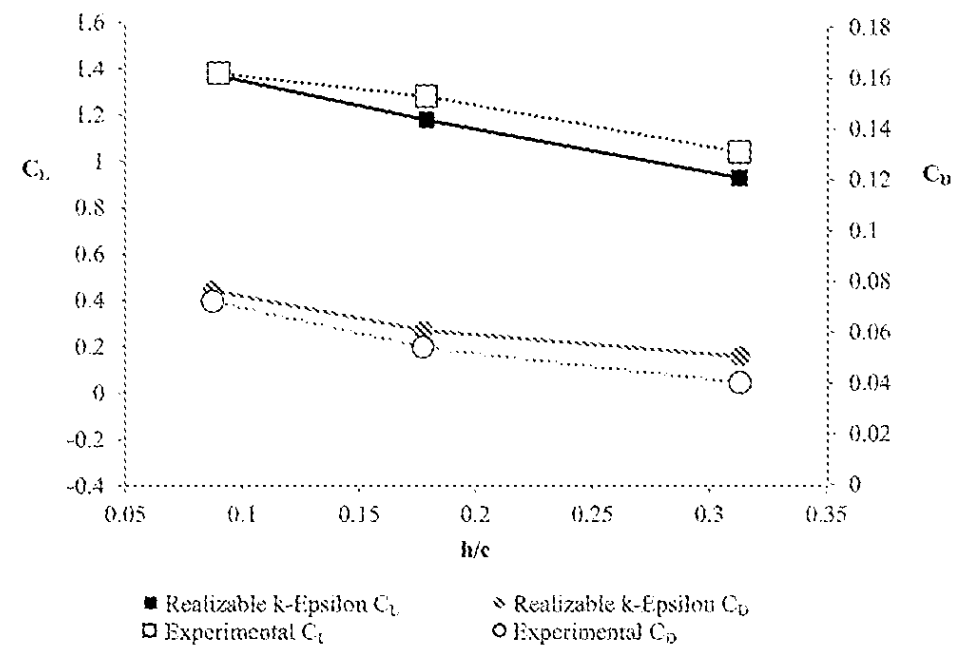

Figure 5. Lift and drag coefficients of the front wing vs reducing height-to-chord ground clearance. 


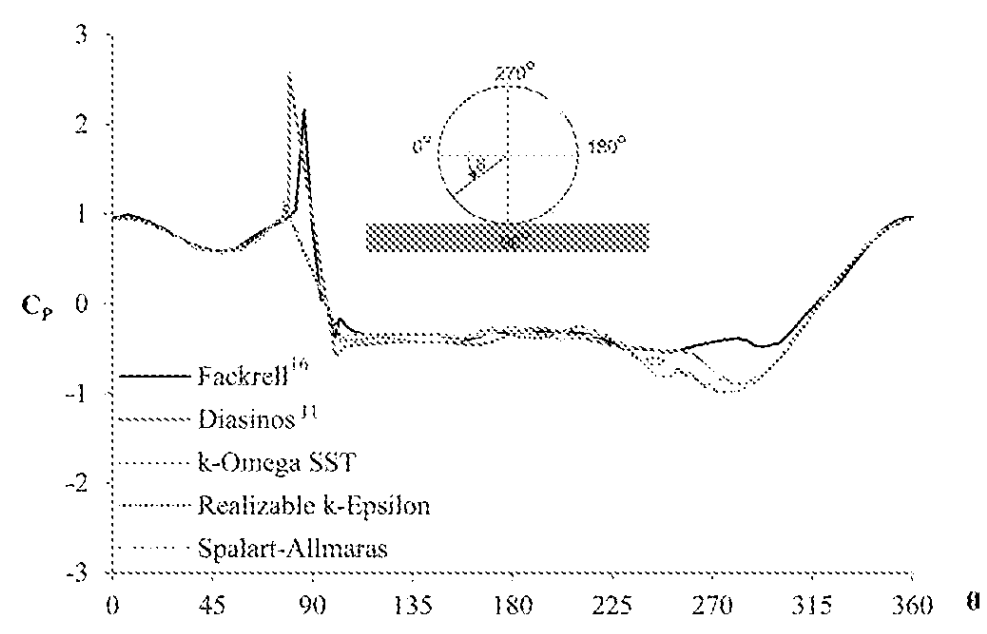

Figure 6. Centre circumferential pressure distributions around the reference wheel (Fackrell) as predicted by different turbulence models.

\subsection{Exposed wheel validation}

A steady-state analysis of an isolated rolling wheel was conducted. Fackrell conducted a series of landmark wind-tunnel tests on a rotating wheel in a wind tunnel, obtained detailed time-averaged surface pressure measurements ${ }^{(16)}$. For the present work the 'A2' wheel was recreated in a facsimile of the wind tunnel and flow conditions used - the wheel features an asymmetric hub and is therefore more complex than the wheel used for the full car study, however a similar mesh was applied. Diasinos was previously able to obtain a relatively accurate steady-state solution for a rotating whee ${ }^{(11)}$, although transient flow effects are certainly important ${ }^{(23-25)}$. The entire surface of the wheel was a rotational boundary with an angular velocity of $89 \cdot 12 \mathrm{rad} / \mathrm{s}$. The wheel had a constant diameter of $415 \mathrm{~mm}$ and a $5 \mathrm{~mm}$ high contact patch step was used. Predicted pressure distributions are shown in Fig. 6.

The large pressure peak occurring where contact is made between the wheel and the ground, identified as the 'jetting region' by Fackrell, was observed using finer meshes not feasible for an entire car - at the selected mesh resolution, this pressure spike is limited to a $C_{P}$ of close to 1 . While this does not affect the rest of the pressure distribution significantly, being highly localised, it does exert a large influence over the predicted lift characteristics of the wheel.

A better match to the experimental results was observed over the front half of the wheel and in the majority of the wake region by all turbulence models. Near the separation point was where the largest discrepancies were observed between results. Fackrell's results predict a separation point noticeably forward on the wheel with a much smaller pressure drop in comparison to the CFD results. The prediction of the separation point has been observed to differ significantly between works in the public domain. Mesh resolution at the boundary had a strong effect towards the location of this point. The results observed for simulations bear a close correlation to the results seen by Diasinos ${ }^{(11)}$, and van den Berg ${ }^{(26)}$, though recent work using finer meshing and an unsteady RANS/DES approach shows better promise in predicting the separation characteristics ${ }^{(25)}$, and wake behaviour observed experimentally ${ }^{(12)}$. The SA and Realisable models predicted a very similar pressure distribution, while the SST model exhibited unsteadiness in separation and as a result was not observed to correlate as closely due to a lack of true convergence attributed to near-wall mesh resolution deficiency. From the results it was decided that either the SA or Realisable k- $\varepsilon$ models would be suitable. The Realisable model was selected overall due to 


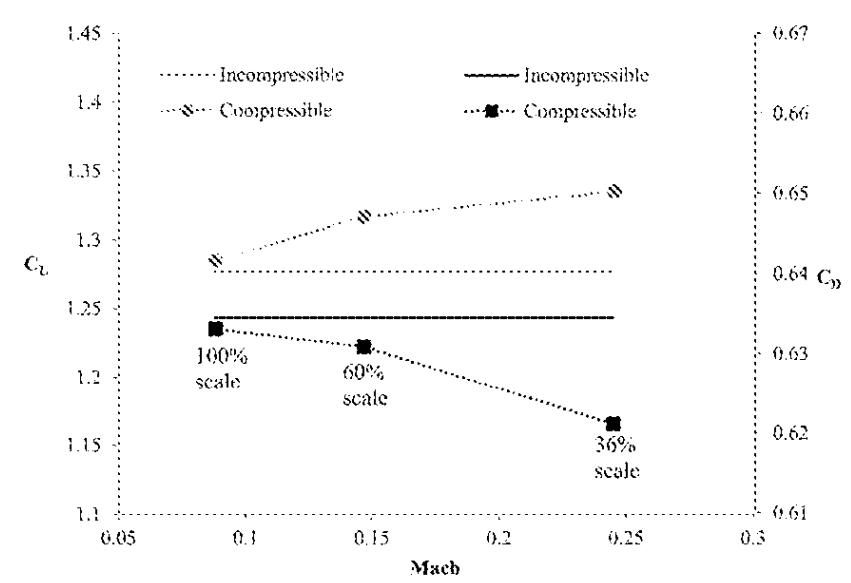

Figure 7. Lift and drag coefficients for the full car as Mach number is increased for a constant Reynolds number.

its anticipated superior properties in predicting the wake region, as observed in other similar studies $^{(11,23)}$, and good performance for the isolated wing.

Table 1

Error and grid convergence indices for aerodynamic coefficients obtained on medium and fine meshes

Parameter

Medium

Fine

$\begin{array}{ccccc} & \text { Error } & \text { GCI } & \text { Error } & \text { GCI } \\ C_{L} & 0.15 & 0.46 & 0.11 & 0.34 \\ C_{D} & 0.08 & 0.24 & 0.06 & 0.18\end{array}$

\subsection{INCREASING MACH NUMBER FOR A CONSTANT REYNOLDS NUMBER}

To best approximate the common scenario of comparing $60 \%$ scale wind-tunnel models to full-scale CFD at the same Reynolds number, a series of compressible and incompressible cases were run for $\mathrm{Re}=9 \cdot 49 \times 10^{6}$ based on car length 1 ; the full scale model at $30 \mathrm{~ms}^{-1}$ (Mach 0.0882), the $60 \%$ scale model at $50 \mathrm{~ms}^{-1}$ (Mach $0 \cdot 1470$ ), and, in an approximation of the scale that might be feasible at a smaller, university-style tunnel, a $36 \%$ model at $83.33 \mathrm{~ms}^{-1}$ (Mach 0.2450 ). The incompressible results were essentially identical ( $0 \cdot 02 \%$ difference) across all cases, indicating that the small changes to mesh $\mathrm{y}^{+}$values were not influential towards the solution.

If the compressible case is assumed to be the best representation of reality, clear trends can be identified from Fig. 7. At the lowest speed tested, the discrepancies are small and could initially be considered within the margin of uncertainty given that the compressible solver is operating at such a low freestream Mach number.

As anticipated, even at $50 \mathrm{~ms}^{-1}$ significant differences can be observed in predictions of both lift and drag for the car between compressible and incompressible cases. A $1.05 \%$ under-prediction in drag and $1.73 \%$ over-prediction in lift were observed at Mach 0.1470 for the incompressible simulation. These trends exaggerate at the $36 \%$ scale with further increased Mach number. The over-prediction in negative lift is observed to increase to $6.68 \%$, with drag being under-predicted by $1.52 \%$, for the incompressible case. 


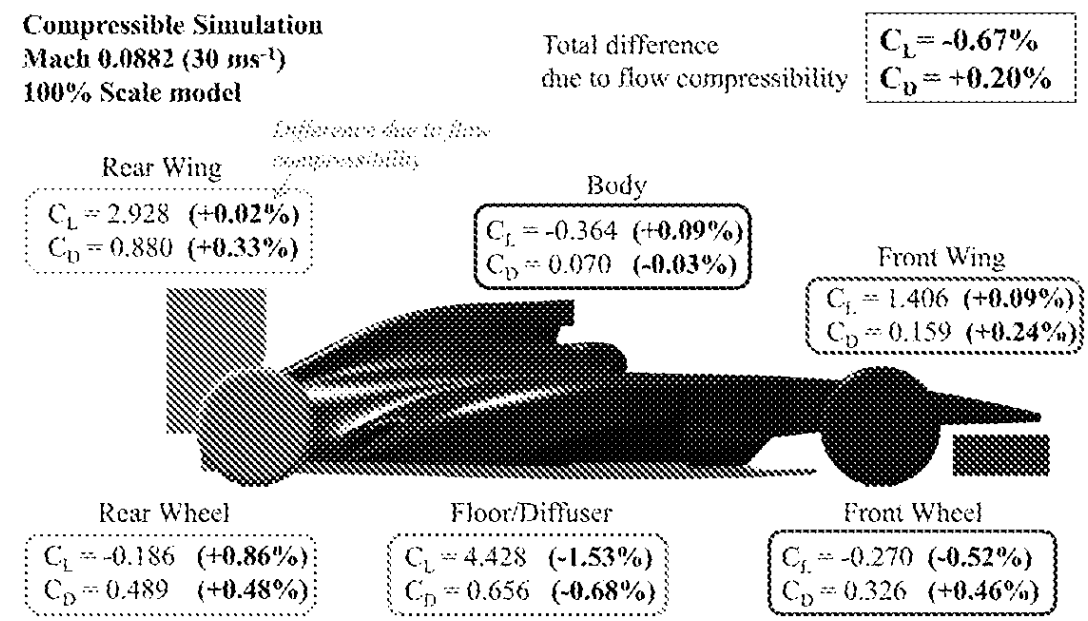

Figure 8. Breakdown of differences in predicted compressible lift and drag coefficients for the major components as compared to the baseline incompressible simulation at Mach 0.0882 .

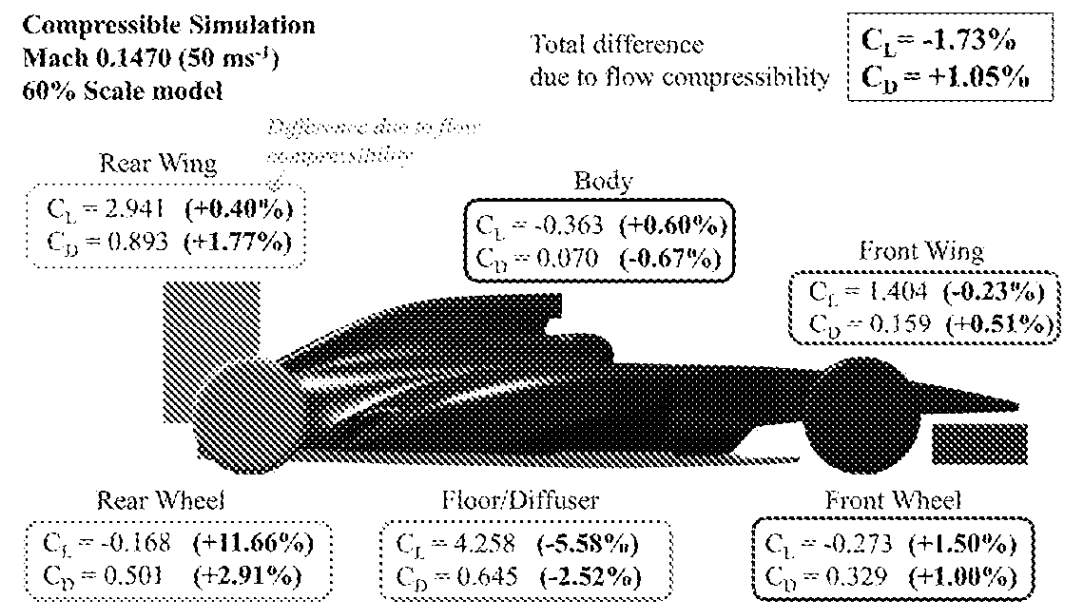

Figure 9. Breakdown of differences in predicted compressible lift and drag coefficients for the major components of the $60 \%$ scale model compared to the baseline incompressible simulation.

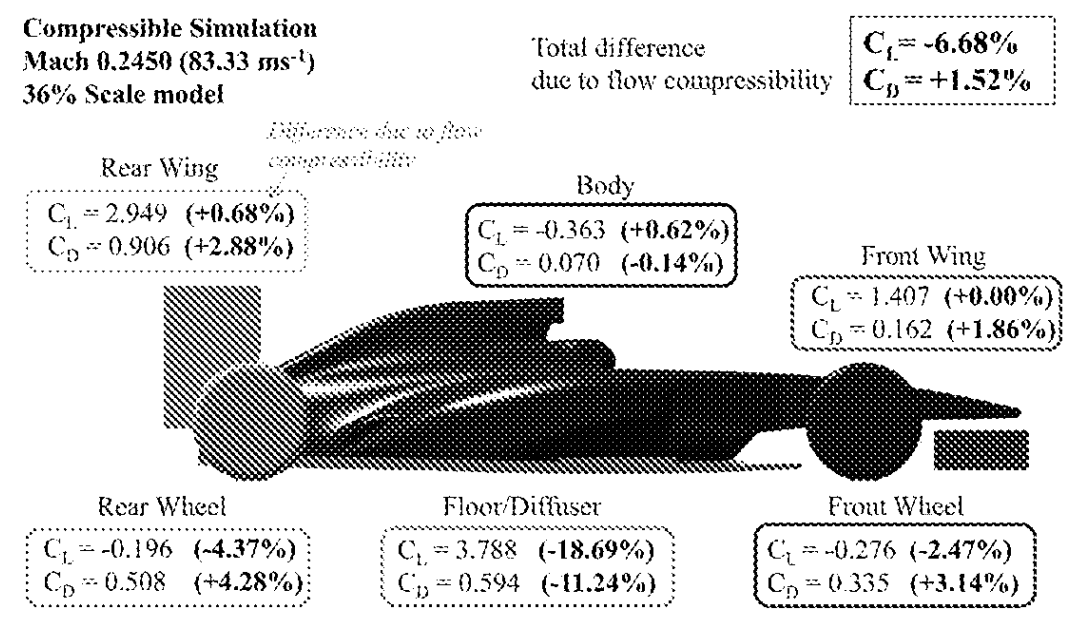

Figure 10. Breakdown of differences in predicted compressible lift and drag coefficients for the major components of the $36 \%$ scale model compared to the baseline incompressible simulation. 
Density changes in the air around the car were observed to increase with increasing Mach number. Table 2 outlines the maximum and minimum relative recorded densities across simulations, showing the continued reduction in density as scale is reduced. Table 2 also indicates that the maximum relative velocity increases with increasing Mach number for the same Reynolds number, further highlighting that scale Mach effects become non-linearly exaggerated as freestream Mach number is incremented. Since high and low density regions are essentially coincident with high and low pressure regions, which dictate the way in which flow accelerates and decelerates around the car, it is clear that the greater the density change, the greater the extent of incompressible under-prediction of the pressure and velocity gradients and therefore the poorer the incompressible force predictions become.

While the differences in lift and drag for the full car - particularly at $50 \mathrm{~ms}^{-1}$ where the windtunnel comparison point would be - may seem small, once they are broken down for the individual components, a more nuanced result emerges, as illustrated in Figs 8, 9 and 10.

Table 2

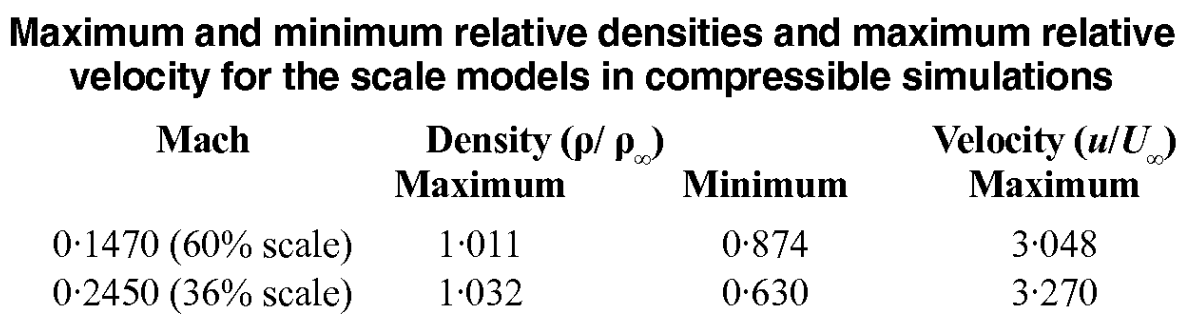

\subsection{Front wing}

Looking first at the front wing, results show differences between the compressible and incompressible predictions were less significant than expected from literature at Mach $0 \cdot 1470$ - less than half a percent for lift and drag, and no reported difference in lift at Mach $0 \cdot 2450$ but with $1 \cdot 86 \%$ more drag in the compressible case. A more detailed examination uncovered that the main element of the wing was making a proportionally larger contribution to the overall downforce for the compressible simulations while the flap was contributing proportionally less - though by approximately half a percent in each case - primarily due to changes in the pressure distribution on the face of the wheel behind the outboard sections.

Sectional pressure distributions are shown at the symmetry plane and at an outboard location of $y / c=1.516$ (in line with the wheel centre) in Fig. 11. At the symmetry plane, where it is a single element, the suction peak in the middle of the wing is greater in the compressible case, causing a $2.00 \%$ higher prediction of sectional $C_{L}$. The opposite occurs at the outboard location, where sectional $C_{L}$ is reduced by $1 \cdot 28 \%$. A wing will produce less downforce in the presence of a wheel due to the adverse high pressure region upstream of the wheel destructively interfering with acceleration of the flow over the lower suction surface of the wing ${ }^{(11,26)}$. A more significant high pressure region was observed in compressible simulations and was found to be responsible for the trend reversal at the outboard section.

The accelerated flow in the mid-section produced a slightly thicker wake due to fractionally earlier separation, resulting in the observed drag increase. While the net effect of these minor plusses and minuses is small, it is clear that the compressible flowfield differences will have consequences for the downstream components, and the wing's aeroelastic and pitching behaviour would be subtly altered too. 

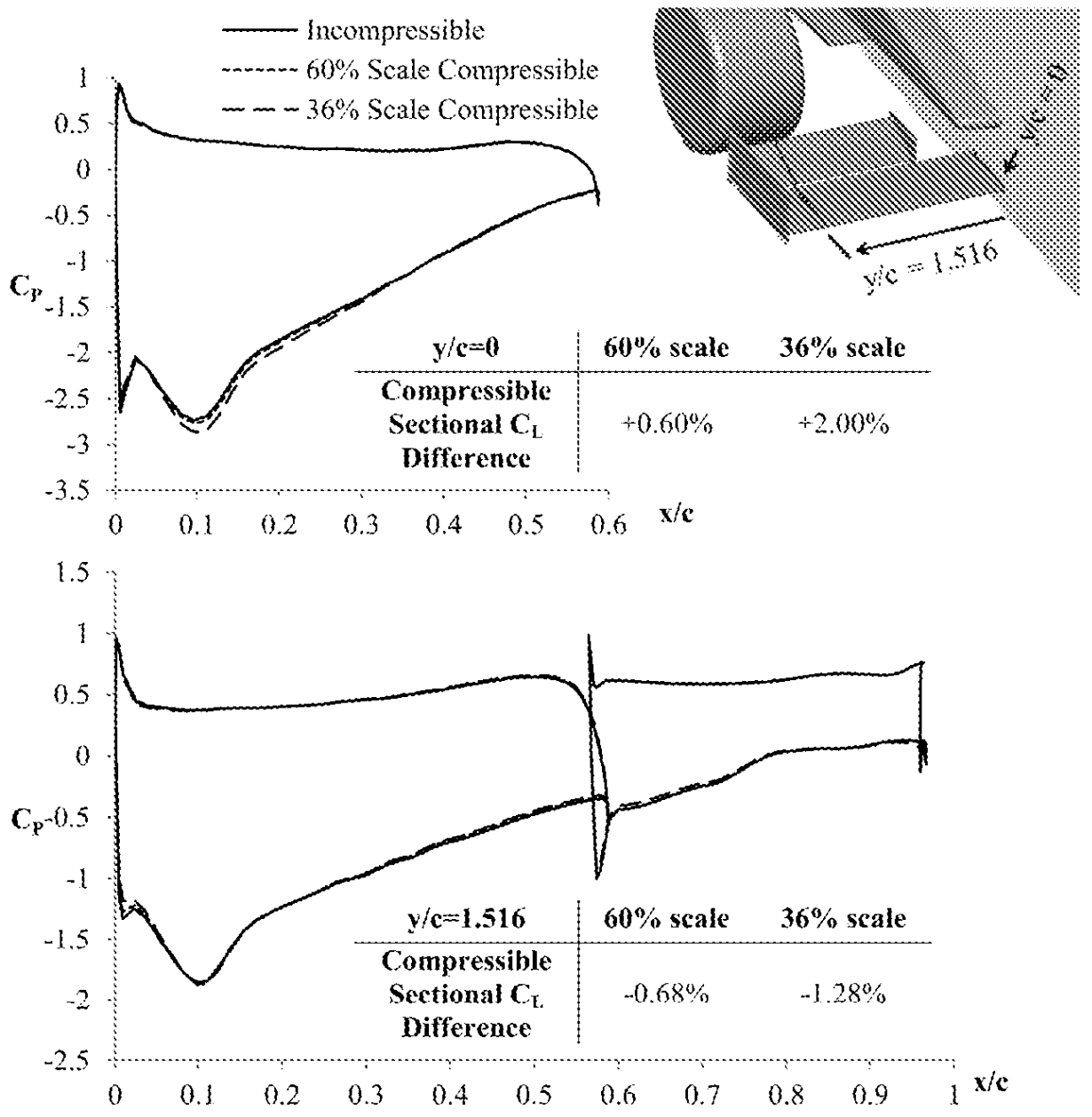

Figure 11. Pressure distributions around both front wing elements at spanwise sections for compressible vs. incompressible predictions at constant Re.

\subsection{Floor/diffuser}

Variation in the negative lift values for the floor and diffuser were observed to be the single most significant contributor to the overall lift differences observed for the car in its entirety. For the $60 \%$ scale case at Mach $0 \cdot 1470$ the incompressible lift prediction was $5 \cdot 58 \%$ greater than the presumably more realistic prediction arising from the compressible case. This alone would be large enough to cause a noticeable discrepancy between an incompressible CFD at full scale to a wind-tunnel simulation at $60 \%$ scale. At $36 \%$ scale the difference grew to $18.69 \%$ in terms of incompressible over-prediction of downforce. With a reduction in downforce, a significant reduction in drag accompanied $-2 \cdot 52 \%$ at $60 \%$ scale and $11 \cdot 24 \%$ at $36 \%$ scale.

Pressure distributions along two longitudinal sections are shown in Fig. 12, where it can be seen that higher pressures were predicted along the rearward half of the floor section for compressible flow, particularly at the highest Mach number. A reduced suction peak at the intersection of the floor with the upswept diffuser section was also observed in all cases. The flow was observed to remain attached in all cases except the 36\% scale compressible case, where the flow was observed to separate in the central diffuser section. The separation was initiated at the leading edge of the strake, as shown in Fig. 13, and also resulted in a loss of the vortex which promoted attached 

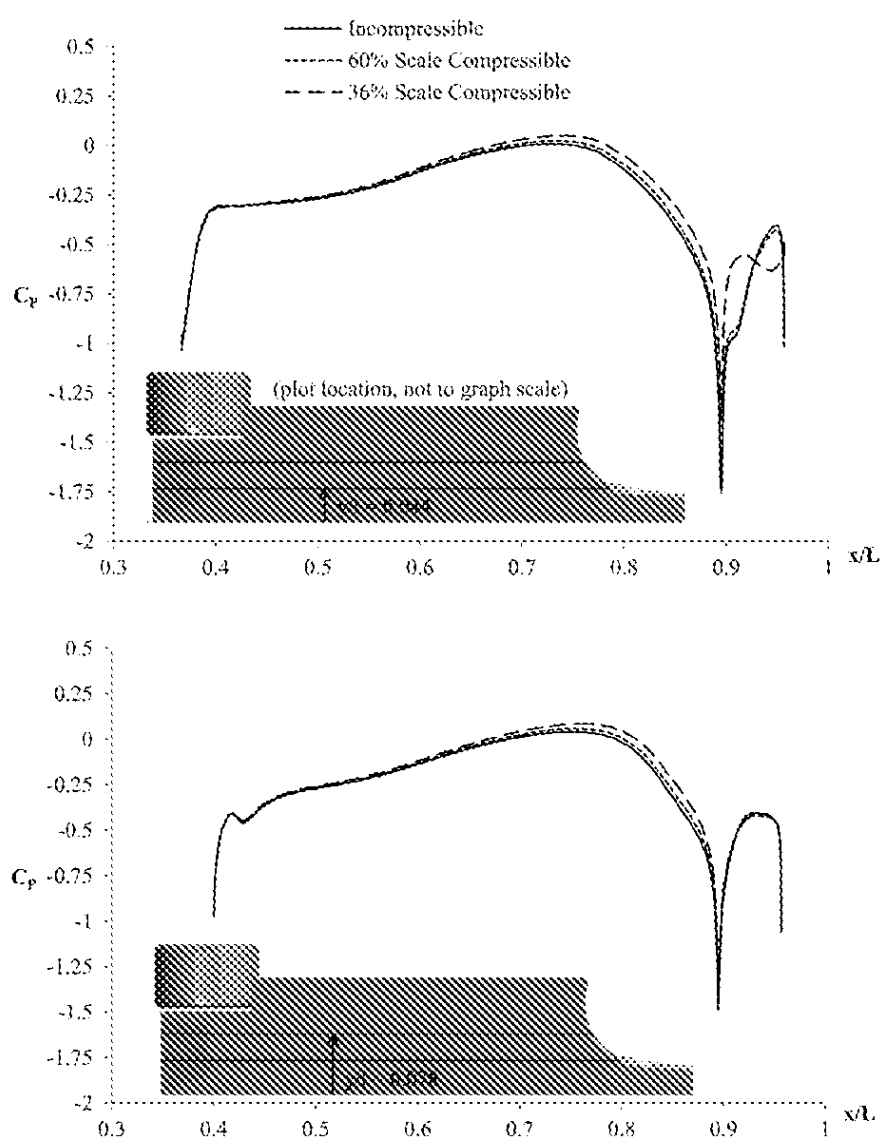

Figure 12. Floor/diffuser pressure distributions at two sections, comparing scale compressible predictions to the baseline incompressible case.

flow for the other cases. This helped explain why the most significant loss of negative lift was observed. The more exaggerated wake was directed wider downstream of the car and had a more significant velocity deficit.

To gain further understanding of this large-magnitude discrepancy, the mass flux exiting the diffuser was measured. The incompressible simulations predicted a notably higher mass flux exiting the diffuser. With increasing Mach number, the percentage of mass passing through the central section fell by around $2 \cdot 36 \%$ and $9 \cdot 59 \%$ through the central section at $60 \%$ and $36 \%$ scale respectively, while the outer channels were affected to a lesser extent. Figure 14 shows the change in mass flux leaving the diffuser, comparing the compressible and incompressible cases. Significant losses are seen to occur due to changes in the flow around the strake. A corresponding increase in mass flux occurred inside the rear wheel for the $60 \%$ scale case. For the $36 \%$ scale case a large increase in mass flux occurred below the strake. Smaller increases occurred between the upper surface of the diffuser and lower rear-wing element, and in the inner half of the lower rear-wheel wake. This reduction in mass flow through the diffuser was a partial cause for the markedly lower production of downforce than the incompressible simulations predicted.

The floor and diffuser have a heavy reliance on vortices, generated both along the length of the floor section and in the rear upswept diffuser section. These promote attachment of the flow and permit increased generation of downforce. Slight changes to the upstream or downstream pressure field could be much more detrimental than would normally be expected. The interaction 
between the diffuser and rear wheel was found to be affected due to compressibility and these changes significantly altered the aerodynamic performance of both components. These effects are discussed further in the following section.

\subsection{Wheels}

Incompressible simulations under-predicted negative lift by $1.50 \%$ and drag by $1.00 \%$ at $60 \%$ scale, and over-predicted negative lift on the front wheel by $2.47 \%$ and under-predicted drag by $3 \cdot 14 \%$ as the scale was reduced further. This was found to be due to the increasing influence of the flow coming off the front wing flap interacting with the wheel and affecting the pressure gradient transition from the stagnation point to the rest of the wheel. This was an extremely local effect, as the rest of the pressure distribution was near-identical around the wheel, including at the separation point near the top of the wheel, which barely changed.

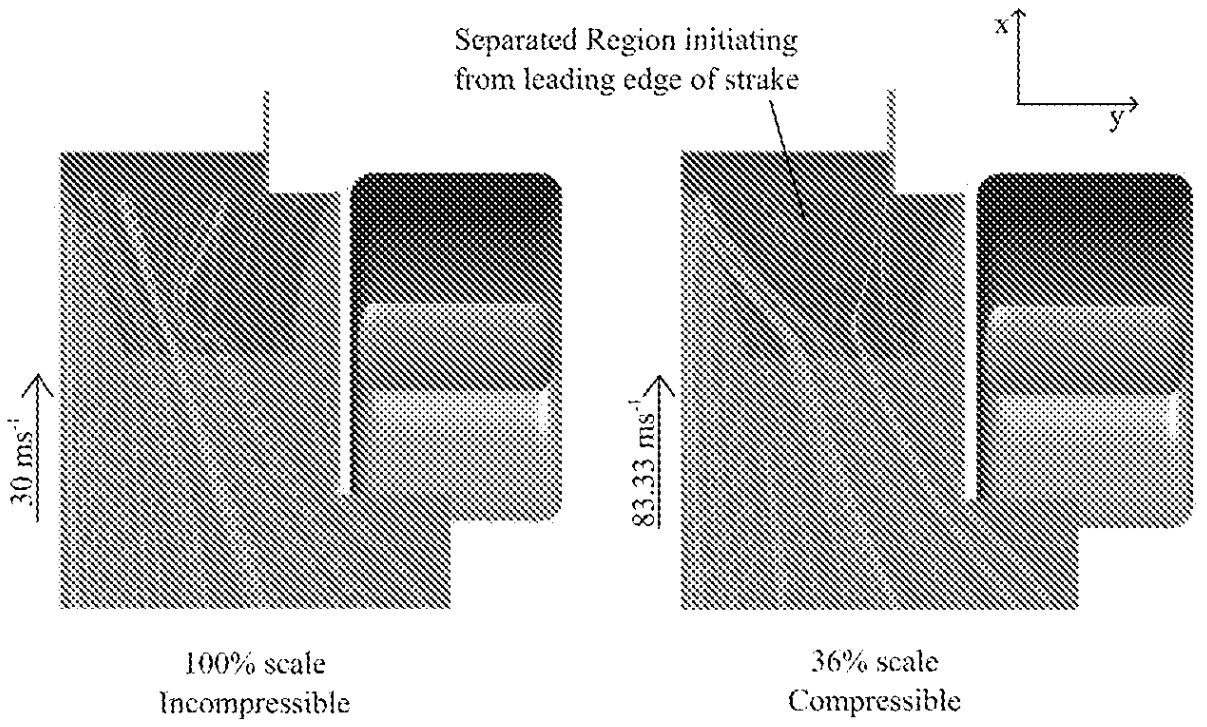

Figure 13. Floor/diffuser wall shear surface streamlines, comparing $36 \%$ scale compressible case to the baseline incompressible case.

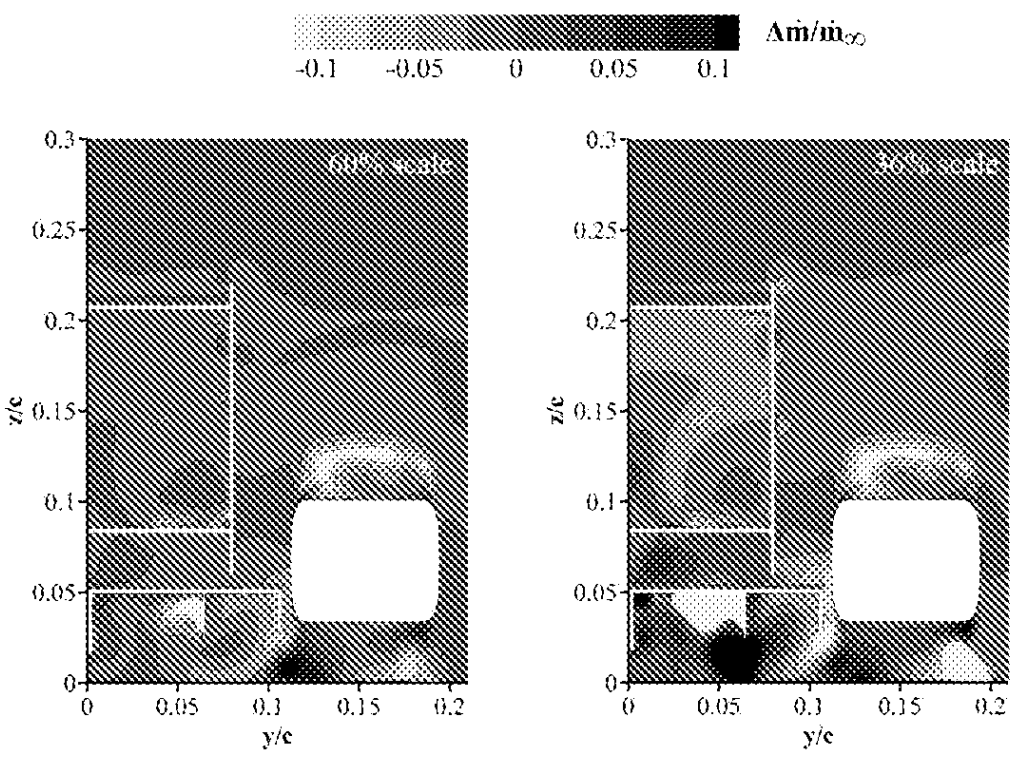

Figure 14. Mass flux distribution difference between compressible and incompressible cases at $x / l=0.956$. 


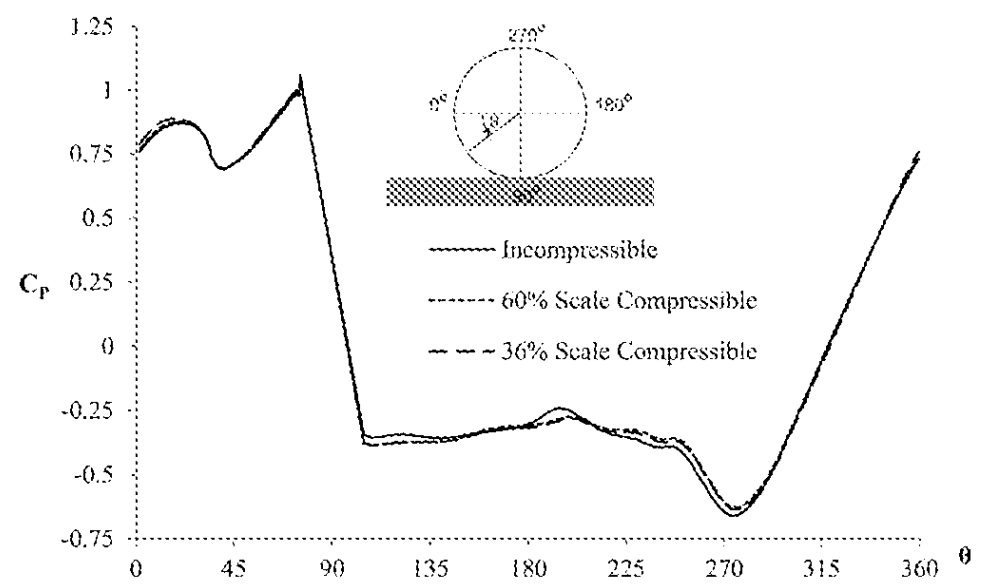

Figure 15. Centre circumferential pressure distributions around the rear wheel, comparing scale compressible predictions to the baseline incompressible case.

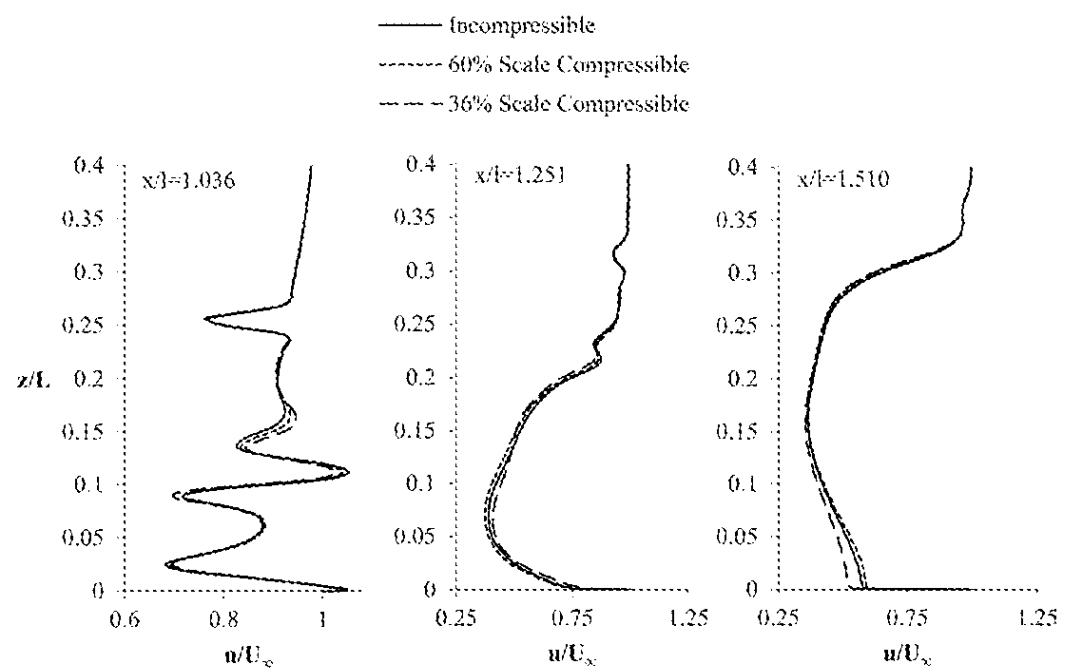

Figure 16. Wake velocity deficits on the symmetry plane at locations downstream of the car for constant Re.

The close interaction of the rear wheels with the diffuser was the single largest cause of discrepancy between compressible and incompressible cases. The rear wheel itself exhibited an $11.66 \%$ increase in negative lift in the compressible case at $60 \%$ scale, but a $4.37 \%$ decrease at $36 \%$ scale. Drag was under-predicted by the incompressible cases by approximately 2.91 and $4.28 \%$ respectively. Different to the front wheel, and shown in Fig. 15, for the centre-circumference section the discrepancies are largely due to a notable change in pressure distribution behind the contact patch and over the rearward portion of the wheel where flow is separated, and indeed at the top separation point itself.

An increase in downstream wake size from the rear wheel was observed with increasing Mach number. A noticeable loss in strength of the lower outboard vortex at higher Mach numbers, as shown in Fig. 14, was observed to result in the rear wheel wake tending to follow a more outboard path. Destructive wheel interference with the rear diffuser section is not an uncommon issue when designing this part of the car. With consideration of compressibility effects it can be seen that this interaction can be much more destructive than was shown by incompressible simulation. The larger high pressure region created upstream of the rear wheel and resultant cross-flow component impeded acceleration of the flow along the length of the floor. This affected the pressure gradient of the flow entering the upswept diffuser section and contributed to the increased pressures along the length of the floor as shown in Fig. 12. This effect was particularly detrimental for the $36 \%$ scale case. 


\subsection{Rear wing}

The negative lift values produced from the rear wing were found to only be minimally affected by compressibility, as can be noted from Figs 8,9 and 10, demonstrating that, away from the presence of the ground, the suction surface low density region was not as significant. At both scales the incompressible over-prediction was less than one percent, and in contrast to the front wing, this difference was consistent along the span of the wing with no section displaying significantly greater discrepancies due to compressibility.

Slightly lower pressures were predicted along the rearward half of the lower suction surface of the main element, following an enhanced suction peak, and the separation point close to the trailing edge moved upstream accordingly, but these differences are barely distinguishable. The fractional increase in separation

drove an increase in drag of $1.77 \%$ and $2.88 \%$ for the compressible cases at $60 \%$ and $36 \%$ scale respectively, compared to the incompressible baseline.

A consistent incompressible under-prediction of pitching moment was also observed in the case of Reynolds scaling. A $1 \cdot 26 \%$ under-prediction occurred at Mach 0.1764 and $1 \cdot 62 \%$ at the $36 \%$ scale.

\subsection{Vortex and wake behaviour}

Primary vortex behaviour for the Reynolds scaling cases demonstrated changes under the different modelling assumptions. For instance, the vortex from the outside of the front wing endplate increased in downstream planar vorticity due to the enhanced wing suction. For the $60 \%$ scale compressible case this increased by $0 \cdot 20 \%$ and the $36 \%$ scale case by $1 \cdot 14 \%$. Despite these changes, the vortex remained on a path that was essentially identical to that predicted by the full scale baseline cases.

Similarly, the strong vortices produced at the rear wing/endplate junction displayed minor changes in behaviour. A slight downwards divergence was observed downstream, and was most obvious for the $36 \%$ scale compressible case. Observed changes in vorticity were negligible. Most significant were the effects to less prominent vortices at the rear wheel and diffuser which affected the close interaction of these components, as discussed earlier.

In the wake region of the whole vehicle, as examined on the symmetry plane, some points of difference could be observed in the Reynolds-scale models compared to the full scale incompressible result, as shown in Fig. 16. Initially very similar across all models close to the rear wing, the $60 \%$ scale simulation at Mach $0 \cdot 1470$ exhibited an enhanced velocity deficit behind the car at the measurement stations of $x / l=1 \cdot 251$, however the $36 \%$ scale simulation predicts this region to feature a reduced deficit. This trend reverses at the final measurement station $x / l=1 \cdot 51$ downstream, with the $36 \%$ scale model predicting a markedly larger velocity deficit across the majority of the wake all the way to the ground plane, which would certainly augment the pressure drag acting on the vehicle. These trends point to the complex interplay of the various components of the car and their relative differences across scales for the same Reynolds number. Between the $60 \%$ and $36 \%$ models the primary difference in qualitative terms was the effect on the lift acting on the rear wheel - which was greater in comparison to the incompressible case at $60 \%$ but less at $36 \%$ - and the wake from the wheels is likely to be the primary driver for the evolving differences in trends seen at the more downstream wake evaluations.

\subsection{Centre of pressure (full car)}

For simplicity, the location of the centre of pressure has just been considered with respect to forces acting in the vertical axis in the present work; this centre is expressed as a percentage of the wheelbase measured from the rear axle-line. A well-designed car will provide a relatively equal 


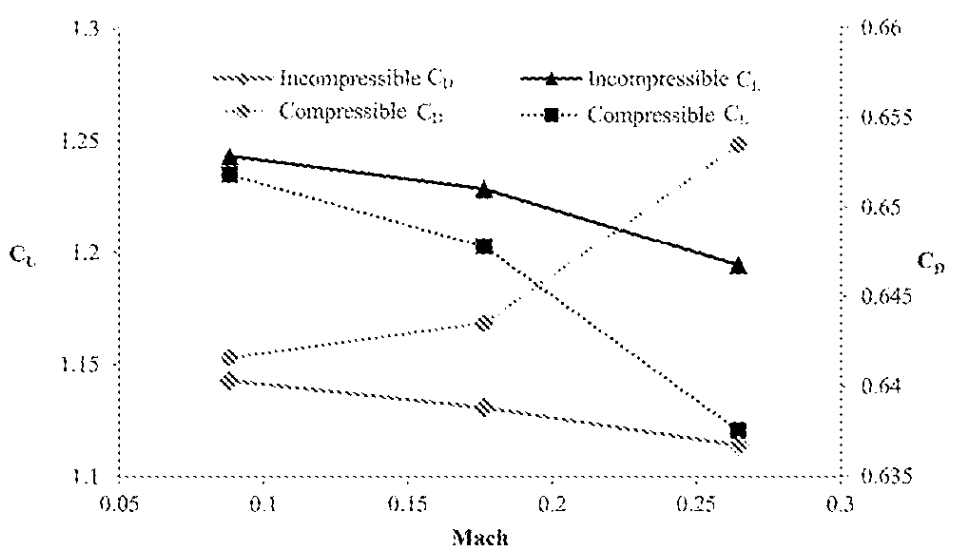

Figure 17. Lift and drag coefficients for the full car as Mach number and Reynolds number increase.

amount of grip at the front and rear wheels. A car with insufficient downforce acting at the rear will have a forward centre of pressure resulting in oversteer, while insufficient downforce at the front will cause the car to understeer.

The Reynolds scaling simulations demonstrated a very minor $0 \cdot 16 \%$ forward shift for the compressible simulation at $60 \%$ scale, but a significant $2 \cdot 23 \%$ shift for the $36 \%$ scale model. A discrepancy between expected design performance and actual on-track behaviour of the order of the latter case would represent an extremely large difference in the anticipated handling of the car; drivers would typically report characteristics as being markedly different if a change of even less than $1 \%$ was implemented from one test to the next, for instance. The forward shift for the compressible simulation is largely due to a loss of rear downforce with a more detrimental interaction between the floor/diffuser and rear wheel, though clearly all components influenced the result to differing but inter-related degrees.

\subsection{INCREASING REYNOLDS AND MACH NUMBER}

In order to determine the effectiveness or otherwise of incompressible simulations for $100 \%$ scale models for general design work emulating real race conditions, rather than pure wind-tunnel correlation, in complement to the cases already run at $30 \mathrm{~ms}^{-1}$ (Mach 0.0882), comparison cases were run at $60 \mathrm{~ms}^{-1}$ (Mach 0.1764) and 90 $\mathrm{ms}^{-1}$ (Mach 0.2646). The latter corresponds to the approximate top speed of a Formula One car at the end of a typical long straight, though some circuits permit faster speeds, and an equivalent Indycar could be expected to top $105 \mathrm{~ms}^{-1}$.

This approach also permits an exploration of the coupling of Reynolds and Mach number in comparison to the cases in which Reynolds number was fixed, and also presents a scenario closer to the extremely challenging task of matching CFD to track testing data.

As can be seen in Fig. 17, the incompressible cases were observed to increasingly over-predict lift in comparison to the compressible simulations, as was observed in the Reynolds-scaled comparisons. At Mach $0 \cdot 2646$ an incompressible over-prediction of $6 \cdot 60 \%$ was observed, compared to $2 \cdot 15 \%$ at Mach $0 \cdot 1764$, in an echo of the trends observed for the previous cases.

The prediction trends for drag were observed to diverge with increasing Mach number, where incompressible simulations predicted a reduction in the drag coefficient for increasing Mach number while the compressible simulations predicted an increase, although the full-car values were relatively minor at $0 \cdot 73 \%$ for Mach $0 \cdot 1764$ and $2 \cdot 57 \%$ at Mach $0 \cdot 2646$. 


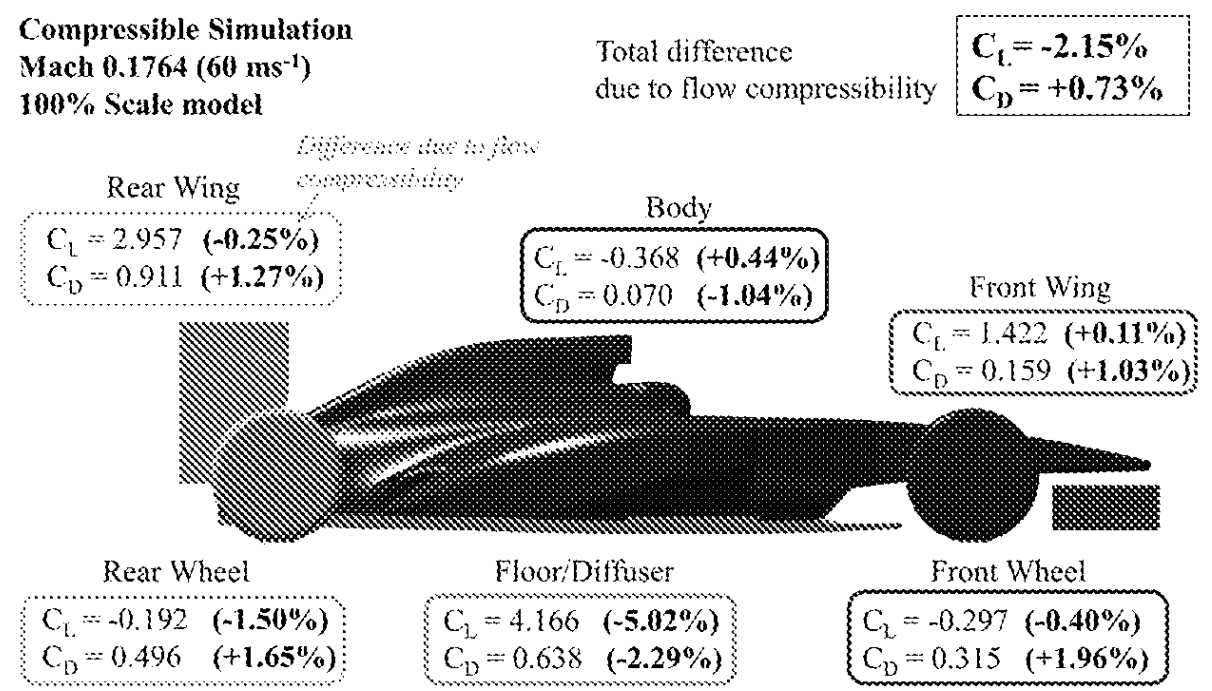

Figure 18. Breakdown of differences in predicted compressible lift and drag coefficients for the major components as compared to the baseline incompressible simulation at Mach $0 \cdot 1764$.

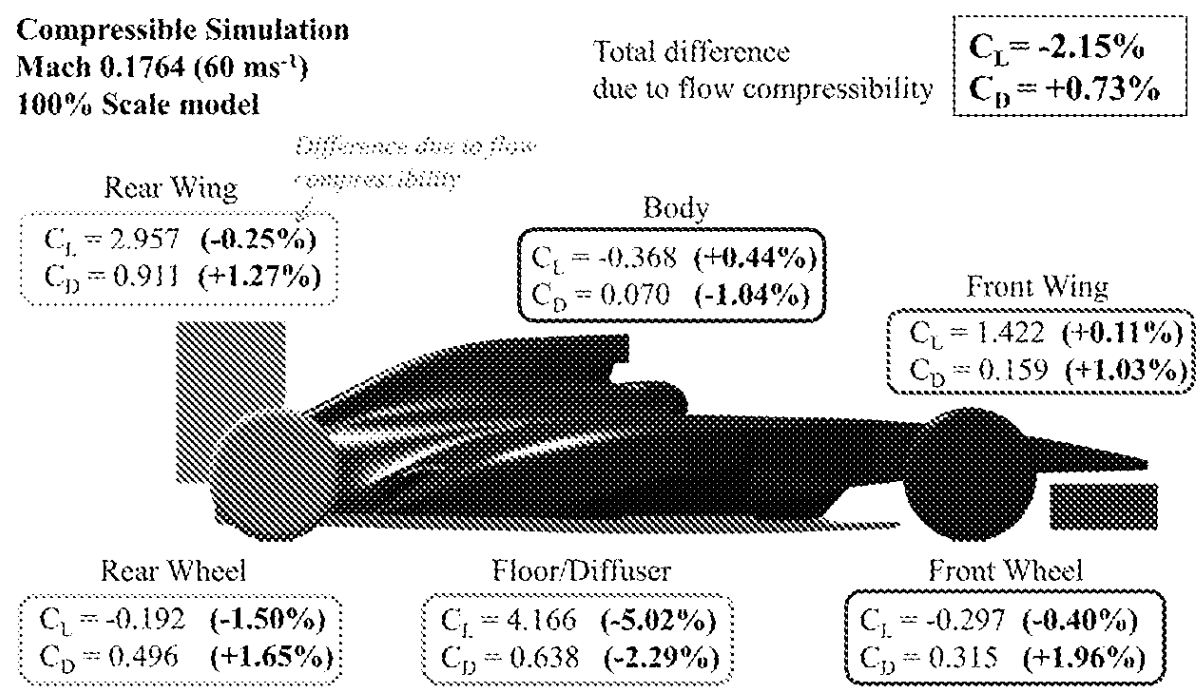

Figure 19. Breakdown of differences in predicted compressible lift and drag coefficients for the major components as compared to the baseline incompressible simulation at Mach 0.2646 .

As noted for the Reynolds-scaled simulations, the whole-car coefficients can be misleading in terms of indicating large local discrepancies between compressible and incompressible cases, and thus each component was again considered in some detail. As could be expected, most of the physical flow features and causes of differences were identical in nature to those already described. However, some notable differences were observed, particularly for the floor/diffuser and rear wheel regions, indicating the overlap of Mach and Reynolds number effects which would prevent any simple compressible correction estimates being applied across the speed range. The component breakdowns are presented in Figs. 18 and 19, with reference also to the Mach 0.0882 case from Fig. 8.

The front wing again does not exhibit any major discrepancies between simulation types, even at Mach 0.2646 where the difference in downforce was $0.51 \%$, and drag was under-predicted by 
$2 \cdot 38 \%$, due to the slightly earlier separation and resultant thicker wake. Unlike in the Re-scaled cases, the compressible simulations predicted marginally more downforce. For this reason, the overall peak acceleration of flow around the front wing relative to the freestream was lower at the highest Mach number, as the front wing began to lose effectiveness in the compressible case compared to the incompressible prediction.

The relative contribution to overall downforce and drag between the main wing and flap element was more exaggerated in this instance. The main element contributed $0.52 \%$ more downforce and $2.42 \%$ more drag towards the overall total at the highest Mach number. This was close to double the difference noted in the Reynolds-scaled case, indicating that a higher Reynolds number affects the front wheel flow more significantly. This is confirmed by the fact that the wheel produces more lift at the higher Reynolds number, with the separation point over the upper surface shifting rearward. The pressure distribution affects the front wing as well as the wheel's own characteristics, though the force coefficient discrepancies are not significantly different.

The floor/diffuser region produced the greatest discrepancies, as in the Reynolds-scaled cases, however the magnitude of the incompressible over-prediction was similar only up until the highest Mach number, where a $-11.93 \%$ difference was seen. At the fixed Re this was $-18.69 \%$ in lift with $11.24 \%$ less drag in the compressible case, whereas at the higher Reynolds number the diffuser features only half a percent difference in drag - the flow remains attached unlike in the lower-Re equivalent, meaning that the diffuser performs more effectively.

Changes in the under-floor and rear wheel interaction as Mach and Reynolds number increased resulted in a more predictable trend of increasing positive lift on the rear wheel. At the highest Mach number the incompressible simulation offered an under-prediction of $20.64 \%$, and a drag over-prediction of $6.71 \%$. The separation point on the rear wheel did not change by any significant amount (whereas the compressible cases showed a marked difference in the Reynolds-scaled simulations), and the wake was drawn more inboard than in the fixed-Re cases due in part to the lower pressure of the relatively better-performing diffuser. The far-field wake was evaluated on the symmetry plane as well; here, the compressible wake was observed to consistently predict an enhanced velocity deficit peak.

Comparing the effects seen on the rear wing to those observed in the Reynolds-scaled case at a similar Mach number, another point of difference was observed, with the compressible cases predicting marginally less downforce, whereas the lower-Re equivalents predicted increasingly more downforce as Mach number was incremented. In the latter cases, a very slight increase in suction on both upper elements was observed, but in the non-fixed Reynolds cases, this suction peak was slightly reduced, as a result of a combination of very subtle upstream differences on the rest of the car, largely driven by the difference in mass flow and resultant pressure underneath the car. As a result, the pitching moment of the rear wing was increasingly under-predicted by the incompressible result, by as much as $1 \cdot 50 \%$ at Mach $0 \cdot 2646$.

As in the Reynolds-scaled instances, increasing Mach number brought with it a forward shift in the overall vehicle centre of pressure when compared to the incompressible equivalent: $0 \cdot 75 \%$ at Mach $0 \cdot 1764$ and 2.66\% at Mach $0 \cdot 2646$. The high Mach incompressible mis-prediction would likely result in a significant difference in anticipated handling of the car.

It is clear that while the general conclusion holds that incompressible simulations offer increasingly and often exponentially different force coefficient predictions, Reynolds number effects also play an important role. However, at the higher end of the Mach numbers examined here, Mach scaling proves to be just as influential as Reynolds-scaling if not more so, and therefore the best accuracy can only be afforded by simulations which match both the Mach and Reynolds number to the application at hand, be it wind tunnel or track conditions. 


\section{Table 3}

\section{Maximum and minimum relative densities and maximum relative velocity for compressible simulations as Mach and Reynolds number increase}

\begin{tabular}{cccc} 
Mach & \multicolumn{2}{c}{$\begin{array}{c}\text { Density }\left(\boldsymbol{\rho} / \boldsymbol{\rho}_{\infty}\right) \\
\text { Maximum }\end{array}$} & $\begin{array}{c}\text { Velocity }\left(\boldsymbol{u} / \boldsymbol{U}_{\infty}\right) \\
\text { Maximum }\end{array}$ \\
$0 \cdot 0882$ & $1 \cdot 005$ & 0.957 & 2.974 \\
$0 \cdot 1764$ & $1 \cdot 017$ & $0 \cdot 818$ & $3 \cdot 127$ \\
0.2646 & 1.038 & 0.703 & $2 \cdot 762$
\end{tabular}

Finally, it is worth mentioning the additional computational expense of running compressible simulations; after all, there would be little hesitation to run a more physically accurate ideal gas-based simulation were it not for the need to solve the energy equation and have the solver compute a more complex flowfield incorporating density changes. In a series of benchmark solutions run in otherwise identical circumstances across 48 processors, the compressible simulations took almost precisely $150 \%$ of the solution time that the incompressible cases did (with a similar number of iterations), and utilised close to $15 \%$ more RAM in the process. While the RAM requirements may not be significant, the additional runtime is clearly an important factor in considering whether the increase in accuracy would be worth the compromise in speed and/or number of configurations able to be tested in any given limited timeframe.

\subsection{CONCLUSION}

A numerical investigation into the influence of compressibility effects around a simplified open-wheel racing car was completed. Results demonstrated that for high-lift aerodynamic designs operating in close ground proximity the effects of compressibility are significant at speeds well below the Mach 0.3 threshold normally applied. Incompressible simulations are generally unsuitable even at Mach $0 \cdot 15$ and below, with pockets of local velocities exceeding three times the freestream value and creating exaggerated low-density, low-pressure flow.

Notably, changes to the interactions between components were the key contributors to some of the most significant observed points of difference. Components operating in the most extreme ground effect were observed to be most affected. For the front wing, incompressible simulations underestimated the extent of flow acceleration and therefore maximum suction. The negative lift produced by the floor and diffuser was found to be the most influenced component; the consideration of compressible flow revealed a more detrimental interaction with the rear wheel and resulted in markedly less predicted negative lift when compared to the incompressible prediction even at Mach $0.0882\left(30 \mathrm{~ms}^{-1}\right)$, but most notably at Mach $0 \cdot 2646$ where discrepancies of over $20 \%$ were observed. The effects towards the behaviour of the prominent vortices off the front and rear wings were small relative to the overall pressure distributions acting on components. Smaller vortices surrounding the rear wheel and diffuser, and general wake velocity deficits were found to be more affected due to compressibility. The car simulated here has a significantly less aerodynamic efficiency compared to modern vehicles, so the estimates presented here are expected to be conservative - actual discrepancies may therefore be greater than those evaluated in this study.

The incompressible assumption is clearly not suitable for racing car aerodynamics if absolute accuracy is a key goal, despite the additional computational expense required to conduct compressible simulations. Compressible simulations become particularly important at Mach numbers of $0 \cdot 15$ and above, and may be one of several factors in poor correlation between wind-tunnel results and incompressible CFD simulations. Observed trends are non-linear and complex due to the close interaction between components. It is clear that a simple compressible correction would not be sufficient. Broadly speaking, 
the results reinforce that any CFD being compared to physical experiments, should reproduce those experiments as closely as is possible and practical in terms of both the geometry and the flow physics.

\section{REFERENCES}

1. Agathangelou, B. and Gascoyne, M. Aerodynamic considerations of a formula one racing car, SAE Technical Paper, 1998, 980399, doi:10.4271/980399.

2. Katz, J. Aerodynamics of race cars, Annu Rev of Fluid Mech, 2006, 38, pp 27-63.

3. Dominy, R.G. Aerodynamics of Grand Prix car, Proceedings of the Institution of Mechanical Engineers, 1992, 206, pp 267- 274.

4. Zhang, X., ToEt, W. and ZeriHan, J. Ground effect aerodynamics of race cars, Applied Mechanics Reviews, 2006, 59, pp 33-49.

5. TOET, W. Aerodynamics and aerodynamic research in Formula 1, Aeronaut J, January $2013,117,(1187)$ pp $1-26$.

6. FIA, 2012 Formula 1 Sporting Regulations, Section 22, Fédération Internationale de l'Automobile. See also URL: http://www.formula1.com/inside_f1/rules_and_regulations/

7. Doig, G., Barber, T.J., LeONARDI E. and NEELY, A.J. The onset of compressibility effects for an inverted aerofoil in ground effect, Aeronaut J, 2007, 111, (1126), pp 797-806.

8. Doig, G., BARBER, T.J. and NeELY, A.J. The influence of compressibility on the aerodynamics of an inverted wing in ground effect, ASME J Fluids Eng, 2011, 133, (6), pp 1-12.

9. ZhANG, X. and ZerHan, J. Aerodynamics of a double-element wing in ground effect, $A I A A J, 2003,41,(6)$, pp 1007-1016.

10. PAKKAM, S.S. High downforce aerodynamics for motorsports, Master's thesis, North Carolina State University, USA, 2011.

11. DiASINOS, S. The Aerodynamic Interaction of a Rotating Wheel and a Downforce Producing Wing in Ground Effect, $\mathrm{PhD}$ thesis, University of New South Wales, Australia, 2009.

12. Saddington, A.J., Knowles, R.D. and Knowles, K. Laser Doppler anemometry measurements in the near-wake of an isolated Formula One wheel, Experiments in Fluids, 2007, 42, (5), pp 671-681.

13. Barber, T.J., Leonardi, E. and ARChER, R.D. Causes for discrepancies in ground effect analyses, Aeronaut $J, 2002,106,(1066)$ pp 653-657.

14. ROACHER, P.J. Quantification of uncertainty in computational fluid dynamics, Annual Review of Fluid Mechanics, 1997, 29, pp 123-160.

15. ZeRIHAN, J. and ZHANG, $X$. Aerodynamics of a single element wing in ground effect, $J$ Aircr, 2000, 37, (6), pp 1058-1064.

16. FACKRELL, J.E. The Aerodynamics of an Isolated Wheel Rotating in Contact with the Ground, PhD thesis, University of London, London, UK, 1975.

17. ZERIHAN, J.An Investigation into the Aerodynamics of Wings in Ground Effect, PhD. Thesis, University of Southampton, School of Engineering Sciences, UK, 2001.

18. Shir, T.H., Liou, W.W., Shabir, A., YANG, Z. and Zhu, J. A new $k$ - $e$ eddy viscosity model for high reynolds number turbulent flows, Computers Fluids, 1995, 24, (3), pp 227-238.

19. MeNTER, F.R. Two-equation eddy-viscosity turbulence models for engineering applications, AIAA J, 1994, 32, (8), pp 69-289.

20. Spalart, P.R. and Allmaras, S.R. A one-equation turbulence model for aerodynamic flows. In AIAA, 30th Aerospace Sciences Meeting and Exhibit, Reno, NV, USA, 6-9 January 1992.

21. DoIG, G. and BARBER, T.J. Considerations for numerical modelling of low aspect ratio inverted wings in ground effect, ALAA J, 2011, 49, pp 2330-2333.

22. Mahon, S. and Zhang, X. Computational analysis of an inverted double-element aerofoil in ground effect, ASME J Fluids Eng, 2006, 128, pp 1172-1180.

23. Mcmanus, J. and Zhang, X. A computational study of the flow around an isolated wheel in contact with ground, J Fluids Eng, 2006, 128, (3) pp 520-530.

24. Axerio-Cilies, J. and IACCARIno, G. An aerodynamic investigation of an isolated rotating Formula 1 wheel assembly, J Fluids Eng, 2012, 134, (12), pp 1-16.

25. Dassanayake, P.R.K., Ramachandran, D., Salati, L., Barber, T.J. and Dotg, G. Unsteady computational simulation of the flow structure of an isolated wheel in contact with the ground, Proceedings of the 18th Australasian Fluid Mechanics Conference, Launceston, Australia, 3-7 Dec 2012.

26. VAn Den Berg, M.A. and Zhang, X. The aerodynamic interaction between an inverted wing and a rotating wheel, J Fluids Eng, 2009, 131, (10), pp 101-114. 